\title{
Intellectual Singularity of Quasi-Holographic Paradigm for a Brain-like Video-Component of Artificial Mind
}

\author{
Yarichin E.M ${ }^{1}$ \\ Brain-like Technology Laboratory \\ (NeocorTek Lab) \\ Krasnoyarsk \\ Russia
}

\author{
Gruznov V.M ${ }^{2}$ \\ Trofimuk Institute of Petroleum \\ Geology and Geophysics, Siberian \\ Branchof Russian Academy of \\ Sciences, Novosibirsk, Russia
}

\author{
Yarichina G.F ${ }^{3}$ \\ Institute for Business Processes and \\ Economic Management \\ Siberian Federal University \\ SFU, Krasnoyarsk, Russia
}

\begin{abstract}
On the basis of a new post-Shannon information approach (quantitative and qualitative together), a hierarchical process of evaluating video-information by an intellectual brainlike video-component of artificial mind is considered. The development of the classical (Shannon's) informational approach to the level of the new (post-Shannon's) informational approach made it possible to formulate an important additional "bonus" in the form of a differential holographic principle (DHP). DHP made it possible to present video information on a dualistic basis, considering its physical and structural components, considered together. Developed an integral quasi-holographic principle (IQHP) is built on the basis of the DHP. However, in contrast to the DHP this principle represents a supra-physical (abstract) principle, uses a long-range action template and is realized instantly (i.e. with an infinitely high speed). In a joint tandem of physical (quantitative) and structural (qualitative) components of video-information evaluation, the structural component is dominant. Due to this, the technology of the video-component of artificial mind based on IQHP always takes the form of an ascending hierarchy of structured (abstract) evaluations of videoinformation. This technology also includes a hierarchy of selflearning stages, thanks to which the constant development of macro-objects of video-information in the form of videothesauruses as high-quality measuring scales is carried out. This maintains the relevance, efficiency, and instantaneousness of the video-component of the artificial mind in evaluation videoinformation. Based on the ideas and principles of a new (postShannon) information approach to evaluation video-information, the structural and functional architecture of the videocomponent of artificial mind built. This architecture is not biologically inspired, but it turned out to be surprisingly exactly coinciding with the known structure of the human neocortex (by the number of levels of the ascending hierarchy, by the presence of a hierarchy in direct and feedback, by the method of structuring and collecting input elementary video-data, etc.). A new theorem for a complete sample of video-data, considered together in physical and structural form, is formulated. The direct version of this theorem corresponds to an ascending hierarchy of video-information evaluations based on IQHP and bundles of video-information's evaluations. The inverse version characterizes the global hierarchical feedback, which takes the form of a descending hierarchy of "service" video-information evaluations.
\end{abstract}

Keywords-Differential holographic principle; video-data; structuring; super-saccades; integral quasi-holographic principle; long-range action; bundles; ascending hierarchy; singularity; video-component; video-thesaurus; video-intelligence; architecture; artificial intelligence; artificial mind; full sampling theorem; descending hierarchy; hierarchical feedback

\section{INTRODUCTION}

The progress of intelligent technologies is based on the evolution of the processes of recognition, perception and understanding of the surrounding material World, ideas about which are formed by joint quantitative (physical) and qualitative (structural) evaluations of the diverse information content of the surrounding World. All this is inextricably linked with the achieved depth of human awareness of the nature of the information of the material World, which in the general case is characterized by strong nonlinearity, and because of this, lack of clarity and non-obvious. In this regard, researchers are tempted to replace the solution of complex nonlinear problems of information analysis and intellectual synthesis of "mental" evaluations of information with a variety of intuitive and heuristic approaches. They are a significant (sometimes fatal) simplification of reality, but at the same time they are often proclaimed the only correct means of its reflection. Generally speaking, the term mental, accepted and widely used in psychology, refers to mental images and processes in a person. Therefore, in machine psychology of artificial mind, to maintain continuity, we will use the same term, but in quotation marks. This means that the considered structure, functions, mental images and processes of artificial mind can be compared with analogous mental processes in humans, but refer they exclusively to machine psychology.

Intuitive and heuristic approaches (including the classical quantitative informational approach of K. Shannon and neural networks), generally speaking, no longer meet the requirements for modern intellectual technologies, introducing significant confusion and thereby limiting their scope. Currently, among the promising intelligent technologies, in the implementation of which human civilization is interested, include the technology of strong (human-like) artificial intelligence, which can be considered as a technology of weak artificial mind as well as technology of artificial superintelligence (superhuman artificial intelligence), which can be considered in the quality of the technology of strong artificial mind. For a meaningful construction of these technologies, a new information approach 
to the technologies of artificial mind is required, which will be adequate to their complex "mental" nature. This will make it possible to consciously switch to the synthesis of not only individual specialized intelligent systems, but also multicomponent intelligent systems of artificial mind (weak and strong).

In the short term, the complete replacement of human natural intelligence with artificial mind in most areas of human society it is not yet conceptually and technologically feasible. As an affordable alternative, it is still possible to consider intelligent technologies based on neural networks, which allow emulating some simple ones the capabilities of human intelligence, for example, in the field of pattern recognition. However, modern technologies based on neural networks do not allow provide emulating the complex capabilities of human intelligence, such as understanding images. Without this, is impossible awareness of reaching a frightening threshold for the development of artificial intelligence, beyond which an acute antagonism of natural and artificial minds can begin, as well as the cataclysm following from this antagonism in the form of a technological singularity (of explosive acceleration of scientific and technological progress and the creation of a super mind as of hypothetical a danger to human civilization). Similar fears about mechanical machines have already occurred in the history of human society, which, nevertheless, was able to create a highly developed civilization using the achievements of machine mechanics.

It is now clear that technologies that are much more complex, sophisticated and abstract than the mechanical technologies of complex machines and mechanisms will be used for the development of human civilization. This process stretched out in time will lead to certain transformations in human society and the emergence of a new civilization, most likely of the information type, based on deep awareness of a new (post-Shannon) informational approach-analysis (quantitative and qualitative together) to the material World around. The natural development of this new informational approach is an intelligent informational approach-synthesis, which, with the help of a bottom-up hierarchical synthesis of the initial physical evaluation of weak information, allows to adequately restore the initial information as a whole. This process can be viewed as a "mental" representation of information from the surrounding material World in the "mental" space of the intellectual system of artificial mind (ISAM). This artificial (virtual) space is induced in the process of internal synthesis in the ISAM of the ascending hierarchy of information evaluations (quantitative and qualitative together) and is a necessary means for their formal mathematical "awareness" (recognition, perception and understanding) by artificial mind.

The existing pessimism regarding the solution of these complex problems at the present time is most likely due to:

- Incorrectness of intuitive and bio-inspired heuristic attempts (for example, based on neural networks) to solve the problem of artificial intelligence as the basis of artificial mind;

- Commercial haste in the development of the first productive, but still "raw" results in the field of artificial intelligence, which were initially poorly understood and, generally speaking, are still largely not fully understood;

- Focusing not on the dominant, but on some secondary properties of artificial intelligence, in other words, not on the understanding of "strong" information, but on the recognition of "weak" information;

- Inadequate approaches to solving the problems of artificial intelligence.

Apparently because of this, many developers of artificial intelligence systems have developed a firm opinion about the existence of a kind of "magic" of intelligent systems, which is still not understood and therefore is not available to the vast majority of modern developers.

It is possible to comprehend and understand this "magic" only with the help of modern fundamental science. At present, on the basis of an objectively expanded concept of information to complete information (quantitative and qualitative together), fundamental science is able to propose new ways to solve the problem of artificial mind, both in small and in general. These solutions can be built on the correct basis, they are more effective and have a clearer perspective than the currently proposed bio-inspired intuitive and heuristic templates for solving artificial intelligence problems.

In this paper, one of the most important intellectual technologies of artificial intelligence, namely, video-intelligent technology, is considered as a hierarchically ascending synthesis of "mental" evaluations of video-information. This process begins with the synthesis of evaluation of weak (1D, 2D) video-information, which have a "rough" topology, and develops towards the synthesis of evaluations of "strong" (3D, 4D) video-information with a "finer" topology. This approach corresponds to the well-known perceptual model of the world in human consciousness, which was proposed in 1935 in the USSR by the physiologist N.A. Bernstein and pointed to the topological concept of the brain, namely, that "the brain reflects the world topologically" [N.A. Bernstein].

In the future, we will proceed from the fact that it is the post-Shannon informational approach, which was verified on the problems of video-information analysis, with the appropriate development, is able to effectively solve the problems of "mental" synthesis of video-information evaluations using the structural (topological, qualitative) concept of brain-like intelligent systems. It should be noted that the processes of natural or artificial formation of videoinformation are significantly different from the processes of constructing "mental" evaluation of video-information (generally speaking, more than formal analysis differs from formal synthesis). Due to this, the new (post-Shannon's) informational approach makes it possible to establish only the most general fundamental requirements for the "mental" synthesis of video-information evaluations. Namely, the evaluations of video-information should be formed as a result of strong "mental" interactions (interactions-measurements). In addition, video-evaluations should be complete and therefore include physical and structural components considered together. Finally, artificial vision, as the most informative 
component of artificial intelligence, should be a sequence of gradually more complex stages of "mental" recognition, perception and understanding, which are considered together in the form of an ascending hierarchy of "weak" (1D, 2D) and of "strong" (3D, 4D) evaluations of video- information. The latter already possess the quality of "mentality" comparable to the mental activity of a person, about which it is known that it is an extremely fast process. This circumstance did not pass unnoticed in human society, the centuries-old folk wisdom of which was reflected in folk tales, where the correct answer to the riddle "What is the fastest in the world?" was the answer "Thought".

Thus, the post-Shannon informational approach-analysis needs to be rethought and supplemented in order to achieve its adequacy in solving the problems of intellectual synthesis of "mental" evaluations of video-information, including by creating an information theory of artificial mind. Of its particular case is the information theory of intelligent artificial vision the sketch of which [1] was discussed earlier and did not have deep theoretical support. It should be noted that the infrastructure of the ISAM includes not only the ascending hierarchy of "mental" evaluations of information (recognition, perception and understanding), which is directed to the "mental" space of the ISAM, but also the descending hierarchy of service transformations in the form of video-information by feedback, which is directed back to the input ISAM. Latent and not obvious due to its nonlinearity "mental" informational nature of video-intelligence sensory component of artificial mind can be fully realized only when using the post-Shannon informational approach and no other. This makes it possible to meaningfully solve the problems of systemic informational synthesis of artificial vision in the form of an ascending hierarchy of effective intelligent structures of videorecognition, video-perception and video-understanding of the spatio-temporal content of the surrounding material world.

\section{DIFFERENTIAL HOLOGRAPHIC PRINCIPLE OF VIDEO- INFORMATION}

Currently, the concept of holography has acquired a wide semantic content, which allows it to be used not only in applied, but also in fundamental science [2, 3], for example, in string theory in the form of a holographic principle. The holographic principle as applied to video-information turned out to be, generally speaking, strongly "disguised", and to determine it, a special study was required, the results of which were not fully comprehended at once. Let us consider this on the example of analyzing the process of forming full information (physical and structural together) $[4,5,6,7]$ or, which is the same, video-information, in a some material system.

The need for effective construction of video-information evaluation in the process of artificial vision requires the involvement of a new (post-Shannon) information approach. In this case, the process of evaluation video-information by artificial vision should also be a set of strong interactions (interactions-measurements), but already of artificial origin. A meaningful division of artificial vision into simpler and, therefore, more accessible for implementation, partial interactions-dimensions as a whole corresponds to the new information approach. However, natural and artificial measuring processes have fundamental differences in terms of their implementation. In the natural process of forming videoinformation at the physical level, only natural physical standards of measured quantities are used, which, due to the supra-physical (abstract, qualitative) nature of the visual process, cannot be used even in the processes of natural vision of highly developed living organisms.

This circumstance gives rise to the need to create artificial measuring standards for evaluation video-information, which cannot be physically extracted from their natural environment, since they are supra-physical and are related to the structural component of video-information evaluation. In this regard, taking care of the construction of such measuring standards is a super task of any highly organized natural organism or artificial system, if their goal is survival and prosperity in the real conditions of the surrounding (often extreme) material World. This super-problem is solved in both cases in the same way and is reduced to the construction of the necessary artificial measuring standards by using appropriate training and/or self-training. These measuring standards can be organized in the form of a hierarchy of qualitative measuring scales of low and high ranks at different levels of the hierarchy of an artificial intelligent system. In particular, the ascending hierarchy of quality measurement scales for artificial vision can be represented by an ascending hierarchy of video-thesauruses. At the highest level of this ascending hierarchy is artificial video-intelligence [8], which can be considered as a videocomponent of the corresponding artificial mind. The carriers of the input physical video-data for the artificial vision technology are weak (in the video-information sense) wave fieldsmediators of different physical nature (optical and non-optical), which provide video-information short-range interaction through the physical transfer of weak video-information in space-time. The physical nature of these intermediary fields is wave-like. Therefore, an input optical or non-optical videoreceiver can provide consistent video-reception using modern ideas [9], for example, based on coherent or incoherent holography. In modern video-technology and in living nature, a binocular video-receiver based on stereoscopy, which can be adequately described in terms of incoherent holography, is widespread. However, such an obvious application of physical holography at a low level of the ISAM hierarchy does not mean at all that the use of holographic ideas at higher levels of the hierarchy of "mental" evaluation of video-information will be just as obvious.

For the post-Shannon information approach, the basic is the principle of identity of the presentation of video-information [4 - 6], which can be expressed by the following identity.

$$
D_{m} t_{n}^{m}=\nabla_{m} T_{n}^{m}
$$

where

$$
t_{n}^{m}=t_{n(E)}^{m}+t_{n(S)}^{m} \quad-\quad \text { video-information in global }
$$

coordinates of Minkowski space in the form of physical $\left(t_{n(E)}^{m}\right)$ and structural $\left(t_{n(S)}^{m}\right)$ components considered together; 
$T_{n(E)}^{m}$ - physical component of video-information in local coordinates of effective Riemannian space-time;

$D_{m}$ - operator of covariant divergence in the metric of the Minkowski space;

$\nabla_{m}$ - operator of covariant divergence in the metric of the Riemannian space-time.

Principle (1) operates in the effective Riemannian spacetime [4 - 6] formed by the product of two subspaces - the Minkowski space and the Riemannian space-time of general form. Equating identity (1) to zero, one can obtain a differential conservation law, from which the concept of video-information as the sum of its physical and structural components, considered together in the global coordinates of the Minkowski space [4 - 6], necessarily follows. However, it turned out that identity (1) has one more important and previously unaccounted for informational "bonus". Namely, expression (1) can be additionally considered as a mathematically accurate and physically correct differential holographic principle (DHP).

Indeed, according to identity (1), the covariant divergence in the Minkowski space from video-information in the form of the sum of its physical and structural components is exactly equal to the covariant divergence in the effective Riemannian space-time from only one, namely, by the physical component of video-information. This ensures the identity of the transformation of video-information from a flat Minkowski space-time into a curved effective Riemannian space-time and vice versa. In other words, if the physical component of the video-information is given in local coordinates on the hypersurface of the effective Riemannian space-time, then, in accordance with identity (1), the corresponding videoinformation can be precisely determined as the sum of its physical and structural components in the global coordinates of the Minkowski space inside the effective hypervolume Riemannian space-time. It should nevertheless be noted that if the physical component of the video-information is given on the hypersurface of a general Riemannian subspace-time, then in the effective Riemannian space-time (the product of the general Riemannian subspace-time and the Minkowski subspace) this same component of video-information is necessarily also a function of global coordinates Minkowski space.

Modern concepts [2,3] of duality and the holographic principle are used in string theory. However, these concepts are largely qualitative and have not yet found an exact mathematical justification for them. Its absence is replaced by an intuitive reference to the duality of a two-dimensional (flat) hologram and a three-dimensional (volume) image reconstructed from it, which, generally speaking, is not entirely correct. In fact, and this was shown even at the initial stages [10] of holography research, the highest quality hologram is not a flat, but a volumetric hologram, which allows recording wavefronts scattered by the observed material surface into the volume of the photographic material. This circumstance makes it possible to adequately reconstruct a three-dimensional image, and not necessarily with the help of a coherent light source.

In string theory (M-theory or modern mathematical physics), the ideas of duality and the holographic principle have found their qualitative confirmation in the macrocosm of black holes. Most of the models that string theorists work with relate to specific spaces and interactions of the macrocosm (Universe), but not the real macrocosm around us. In other words, modern string theory uses the holographic principle at a qualitative (intuitive) level and therefore cannot serve as a guiding theory for the problems of assessing video-information in the material macrocosm. This world, as shown earlier in [4 6], is most justified to consider (in relation to the problems of formation and evaluation of information, but not only to them) immersed in the effective Riemannian space-time.

Taking this into account, we will assume that nature naturally makes it possible to dually present video information in the effective Riemannian space-time of the surrounding macrocosm. Both of these approaches are theoretically equivalent and are dually juxtaposed to the same reality (material object or process). These dual video-information theories are identical in their end result to each other, which leads to predictable consequences. Namely, the "hard" information computations in one subspace of the effective Riemannian space-time can be correctly replaced with "easier" information computations in its other subspace and vice versa.

General reasoning is also possible, for example, for an adequate video informational description of the macrocosm that surrounds us, video-information at its external "boundaries" is sufficient. At the same time, it is reasonable to believe that it is the surface, not the volume, that is the place where fundamental processes that generate video-information take place (for example, due to scattering or rereflection of the activating wave field). Then, if the structural component of the video-information is given in local coordinates on the hypersurface of the effective Riemannian space-time, then the DHP (1) speaks of how the video-information in the differential form will mathematically accurately look in the global coordinates of the hypervolume of the effective Riemannian space-time.

The new (post-Shannon) informational approach is based on the metric approach [6], which makes it possible to formulate DHP (1) in a general form physically and mathematically. This principle in the weak-field approximation $\left(D_{m} \rightarrow \partial_{m}, \nabla_{m} \rightarrow D_{m}\right)$ is transformed into a weak DHP, which can be written in the form.

$$
\partial_{m}{ }_{n}^{(0)}=D_{m} \stackrel{(0)}{T}_{n(E)}^{m}
$$

where

(0)

$t_{n}^{m}$ - weak video-information in global coordinates of Minkowski space; 
(0)

$T_{n(E)}^{m}$ - the physical component of weak video-information in local coordinates of an effective Riemannian space of constant curvature, which, due to this, can be considered as a conformally pseudo-Euclidean space;

$\partial_{m}$ - differentiation operator in Minkowski space;

$D_{m}$ - operator of covariant divergence with respect to the metric of the Minkowski space in a Riemannian space-time of constant curvature (or, otherwise, in a conformally pseudoEuclidean space).

The post-Shannon informational approach is based on the existence of differential conservation laws. In particular, the law of conservation of video-information can be written in accordance with identity (1), in the form.

$D_{m} t_{n}^{m}=\nabla_{m} T_{n(E)}^{m}=0$

Based on expression (3), video-information can be defined as the sum of its physical and structural components, considered together in the global coordinates of the Minkowski space.

$D_{m} t_{n}^{m}=D_{m}\left(t_{n(E)}^{m}+t_{n(S)}^{m}\right)=0$

$t_{n}^{m}=t_{n(E)}^{m}+t_{n(S)}^{m}$

If we carefully consider equality (3), then we can see that there is no expression for the structural component of videoinformation in local coordinates. This happens because this component of video-information induces Riemannian spacetime, in which only the physical component of videoinformation manifests itself locally. In this case, the expression $\nabla_{m} T_{n(E)}^{m}=0$ has the form of a differential covariant conservation equation in local coordinates of the effective Riemannian space-time, but does not guarantee the possibility of obtaining the corresponding integral conservation law and, therefore, does not allow us to understand what is conserved in this case. Therefore, this expression does not contribute to the formal definition of the concept of video-information and, in accordance with the DHP (1), indicates that the capabilities of the mathematical apparatus of video-information computations in subspaces-factors of the effective Riemannian space-time differ significantly.

In the hypervolume of the effective Riemannian spacetime, the results of video-information calculations can be globally presented in a evidentform, and their meaning is usually obvious. On the hypersurface of the effective Riemannian space-time, the possibilities of information computations are wider, but due to their local nature, they are much more abstract and non-obvious, and in terms of meaning, they may need a detailed interpretation.

It should be noted that the structural (qualitative) and physical (quantitative) components of video-information, due to DHP, always cross-strongly interact with each other [6]. The activating component of this interaction is the structural component of video-information $t_{n(S)}^{m}$, which can be considered as a "primary" component, and the passive (activated) and therefore "secondary" component is the physical component of video-information $t_{n(E)}^{m}$. These fundamental interdependencies [6] for general videoinformation components, as well as for weak videoinformation, are as follows.

$G_{m n}^{q} T_{q(E)}^{n}=-D_{n} t_{m(S)}^{n}$

$\gamma_{m n}^{q} \stackrel{(0)}{T_{q(E)}^{n}}=-\partial_{n}{\stackrel{(* 0)}{t_{m(S)}^{n}}}^{n}$

where

$G_{m n}^{q}, \gamma_{m n}^{q}$ - tensor of the third rank with respect to general coordinate transformations and connectivity (Christoffel symbol) in Minkowski space;

$T_{q(E)}^{n}, T_{q(E)}^{n}-$ physical component video-information of general appearance and weak video-information;

$D_{n}, \partial_{n}-$ operators of covariant derivative and ordinary partial derivative in Minkowski space;

$(*) \quad(* 0)$

$t_{m(S)}^{n}, t_{m(S)}^{n}-$ structural components (in canonical form) of general video-information and weak video information in global coordinates of Minkowski space.

In accordance with the DHP, the structural component of video-information in local coordinates of the effective Riemannian space-time is excluded from consideration. At the same time, its energy induces this curved space-time and activates the physical component of video-information in it. In the global coordinates of the effective Riemannian space-time, both components of the video-information jointly form, on the basis of their composition, video-information of a general form, which, in accordance with the conservation law, becomes the source of the video-information field of the corresponding physical nature.

\section{Structuring AND COLLECTING VideO-DAtA AT A LOW LEVEL ISAM HIERARCHIES}

Video-information in physical space-time and evaluation of video-information in the "mental" space of the ISAM should support the isomorphism of the second kind [8], in which the relationship between the elements of video-information evaluation in the "mental" space of the system will be the same as between the elements video-information in the real world. Due to this, artificial vision can be considered as a process of mapping the visually available properties of the surrounding material world into their identical virtual representation in the "mental" space of ISAM. This is a necessary condition for the correct evaluation of video-information about the current space-time environment. The properties of video-information (physical and structural together) can only be virtually 
emulated by the properties of video-information evaluation in the "mental" space of ISAM. Due to this, the evaluation of video-information can be considered as a result of the calibration (supra-coordinate) transformation of videoinformation. However, it turned out to be difficult to apply the calibration ideology for direct reformulation of videoinformation into a supra-coordinate form of video- information evaluation. Indeed, the process of evaluation video-information in ISAM is an ascending hierarchical process that is directed from evaluations of weak video-information to evaluations of strong video-information, and this is fundamentally different from the natural process of forming video-information in nature. The formation of video-information proceeds from physically inaccessible strong general video-information to available weak video-information, i.e. top-down. Generally speaking, video-information in nature is formed as strong video information with a "thin" topology. At the same time, in the real World, the transfer of strong video-information in spacetime is possible only by its reduction into weak videoinformation and is carried out by a weak video-information field, the source of which, in accordance with the conservation law, will be the corresponding weak video-information.

Thus, the input video-signal of the ISAM is always a weak video-information field of one or another physical nature, which carries out a calibration supra-coordinate transformation [8] and has nothing to do with coordinate transformations. It is because of this that there is a second-order isomorphism (identity) of the evaluation of weak video-information generated by ISAM and weak video-information, which is considered as a source of a weak video-information field.

In the macrocosm surrounding us, video-information is usually viewed in terms of intensity. Therefore, the evaluation of the intensity of weak video-information can be written, neglecting the difference between the covariant and contravariant tensor components in flat space-time, as follows.

$$
\begin{aligned}
& \stackrel{\hat{(0)}}{I^{m n}}=(\hat{(\hat{0})})^{m n}=\left(\begin{array}{cc}
\hat{(0)} & \hat{(0)} \\
t_{(E)}^{m n} & +t_{(S)}^{m n}
\end{array}\right)^{2}=
\end{aligned}
$$

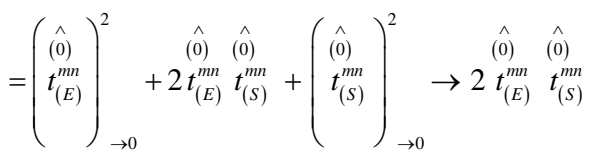

The full quantum of video-data (FQV) can be written (using the notation [8]) in the form

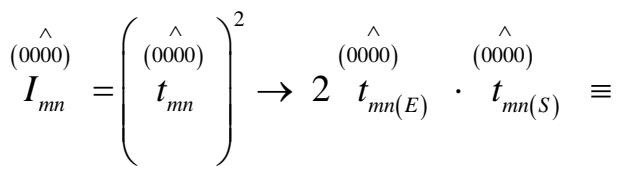

$$
\begin{aligned}
& \equiv{\stackrel{(0000)}{t_{m n(E)}}}^{(\hat{0000)}} \cdot d \hat{t}_{m n(E)}=\omega_{0} \omega_{1}
\end{aligned}
$$

where
$(0000)$

$t_{m n(E)}=\omega_{0}$ - physical quantum of weak video-information evaluation (local physical sampling in the form of a scalar, which can be considered as a differential 0-form);

$$
d \hat{t}_{m n(E)}^{(\hat{0000)}}=d \omega_{0}=\omega_{1} \text { - structural quantum (local 1-qualt) of }
$$
evaluation weak video- information, which can be considered as a differential 1-form.

Expression (8) formally corresponds to a hologram formed by the interaction of two partially coherent processes, which take the form of physical (partially physicalized) and structural (partially geometrized) components of weak videoinformation. In this case, the physical component of weak video-information "delegates" to expression (9) for FQV a physical quantum (physical sample in the form of 0-form), and the structural component of weak video-information, respectively, a structural quantum (local quality in the form of 1 -form). Considering the existing experience in processing holographic video-data, it is possible to consider the question of the relative importance of the physical and structural components of the input weak video-information of the ISAM.

Structural reformatting (structuring) of physical video data is carried out in ISAM using FQV and artificial saccades, the purpose of which is similar to the purpose of saccades of natural vision. Artificial saccades can be represented by a linear combination of FQV.

$$
C_{1, i(t)}=\sum_{i=1}^{I} \omega_{0, i(t)} \omega_{1, i(t)} \rightarrow \bigcup_{i=1}^{I} \omega_{0, i(t)} \omega_{1, i(t)}, i=\overrightarrow{1, I}
$$

Here $C_{1, i(t)}$ - his is an artificial saccade formed by a dynamic linear combination of neighboring FQVs, which is equivalent to their sequential scanning, considering the local spatial orientation of each FQV included in this linear combination.

In the future, these artificial saccades form a certain set $I$ in which each artificial saccade has a small 1D local spatial size. This allows us to consider artificial saccades as elementary "dimensional" dynamic structures. Therefore, combining artificial saccades into compound linear combinations (super-saccades) will dynamically "measure" 1D video-data of a much larger size. Such $1 \mathrm{D}$ video-data can be considered as a set $J$ of super-saccades, which is much smaller than the set of original artificial saccades $I$. Thus, in the process of 1D learning (self-learning), super-saccades perform the function of some intermediate dimensional 1D video-data, which can later be used to construct a 1D video-thesaurus (scale of dimensional 1D video-data) of ISAM.

$$
C_{1, i(t) j(t)}=\sum_{j=1}^{J}\left(C_{1, i(t)}\right)_{j(t)} \rightarrow \bigcup_{j=1}^{J}\left(C_{1, i(t)}\right)_{j(t)}, j=\overrightarrow{1, J}
$$

If the time interval $t_{i}=T_{I} / I$ determines the duration of a certain $i$ current "artificial" saccade without considering the protective scanning time intervals, then the time interval 
$t_{j}=T_{J} / J$ determines the duration of a $j$ certain current super saccade (intermediate 1D video-data). In this case, expression (11) for intermediate 1D video-data synthesized by artificial saccades and super saccades will be fully defined in 1D space. This dynamic set of intermediate $1 \mathrm{D}$ video-data generated over time $T_{i j}=T_{I} \cdot T_{J}\left(T_{I}=t_{i} \cdot I, T_{J}=t_{j} \cdot J\right)$ forms a $1 \mathrm{D}$ videothesaurus $C_{1, j}\left(C_{1, i}\right)$.

$$
\begin{gathered}
C_{1, j}\left(C_{1, i}\right)=\sum_{j-1}^{J}\left(\sum_{i=1}^{I} \omega_{0, i} \omega_{1, i}\right)_{j} \rightarrow \\
\rightarrow \bigcup_{j=1}^{J}\left(\bigcup_{i=1}^{I} \omega_{0, i} \omega_{0, j}\right)_{j}, i=\overline{1, I}, j=\overline{1, J}
\end{gathered}
$$

where

$$
C_{1, j}\left(C_{1, i}\right) \quad-\quad 1 \mathrm{D} \quad \text { video-thesaurus, dynamically }
$$

replenishing due to previously unknown current 1D intermediate video-data, qualitatively presented by splitting into "dimensional" artificial saccades and super-saccades;

$$
\boldsymbol{J} \cdot \boldsymbol{I}=\boldsymbol{K}-\text { the total number of } 1 \mathrm{D} \text { video-data }
$$
accumulated in the $1 \mathrm{D}$ video-thesaurus.

The strings metaphor [2, 3], [11 - 13] in ISAM is that the relationship between the FQVs and global 1-, 2-, 3-, 4-kvalts in the macrocosm can be considered similarly to the relations between strings and branes in the microcosm. In this case, the FQV can serve as an analogue of a strings, and the global 1qualts can be considered as a 1-brane. Branes possessing one, two, three or four dimensions [11 - 13] and can be considered as "layers" of space or as "layers" covering the corresponding space. The dimension of a brane is the number of its dimensions (degrees of freedom) possessed by objects that are "captured" or, in other words, associated with the brane.

However, string theory (like quantum theory) is still a theory of the microworld and in an explicit form cannot be used as a productive theory for evaluating video-information in the macroworld. In fact, the string metaphor in ISAM predicts the complexity and multistage solution of the problem of "understanding" video-information, considered as a complex problem of joint evaluation of video-information in small (evaluation weak 1D and 2D video-information) and in general (evaluation "strong" 3D and 4D video-information).

Reformatting into a structural format is carried out on the basis of long-range measurements supported by structured (abstract) video-information macro-objects (in the form of 1D, 2D, 3D and 4D video- thesaurus), which have the properties of structural (qualitative) measuring scales. In the simplest case, these measuring scales can be constructed by software and hardware (for example, in the form of a structural protothesaurus), but in the general case, only by training / selflearning ISAM. In this case, the well-known topological property [14] is used, according to which a structural videoinformation macro-object of dimension $N$ can be accurately represented by a set of dimensional video-structures of dimension $N-1$ that divide this initial macro-object of dimension $N$ into arbitrarily small parts, and this cannot be done using a set of dimensional dimension video-structures $N-2$.

The physical and structural components of weak videoinformation are connected by identity (13), which characterizes their deep interconnection and can be represented as the following schematic expression.

$$
\stackrel{(0)}{T_{(E) q}^{n} \stackrel{d}{\rightarrow} \gamma_{m n}^{q} \stackrel{(0)}{T_{(E) q}^{n}}=-\partial_{n} \stackrel{(0)}{n}_{(S) m}^{n} \stackrel{\delta}{\leftarrow} \stackrel{(* 0)}{\leftarrow} t_{(S) m}^{n}}
$$

where

$\stackrel{(0)}{T_{(E) q}^{n}}=\stackrel{(0)}{t_{(E) q}^{n}}$ - local meaning (structureless physical sampling, differential 0-form, 0-qualt) of the physical component of weak video-information;

$$
t_{(S) m}^{(* 0)} \text { - the structural component of weak video-information }
$$

in the canonical form (in accordance with identity (13), it can be considered as a differential 2-form);

$d-$ an outer differentiation operator (coboundary operator or, in other words, a cohomological operator) that increases the order of a differential form by one and coincides (in the case of an outer differentiation of 0 -forms) with the ordinary differentiation operator;

$\partial$ - boundary operator dual to the operator $d$ and defining the homology group dual to the cohomology group;

$\delta$ - the codifferentiation operator conjugates to $d$ and decreasing the order of the differential form by one (the divergence [15] of the skew-symmetric tensor).

Expression (13) differs from the original identity (7) in that its left and right sides are supplemented, respectively, by the operators of external differentiation $d$ and the operator of codifferentiation conjugated to it. These operators respectively increase and decrease the order of differential forms by one for 0 - and 2-forms of weak video information. In this case, the left and right sides of identity (13) form one and the same quantity, namely, a differential 1-form, which can be compared to a structural quantum in the form of a local quality. From expression (13) it follows that the 2-form of the structural component of weak video information, activates and thereby completely determines its 1 -form, in which the carrier of local structural properties (local orientation) is the local qualt.

The analysis of the identity (13) allows us to conclude that local quantities can be built by differentiating the corresponding physical samples of weak video- information evaluation. In fact, this can be considered as the simplest inverse problem of a low hierarchy level with a solution algorithm represented by expression (13). The result of this solution is the construction of a set of structural quanta (local qalts) for the subsequent evaluation of their spatial orientation and structural reformatting. Further, full quanta of video-data (FQV) are formed, which are jointly considered the corresponding physical and structural quanta of the evaluation 
of weak video-information. This makes it possible to construct a linear combination of FQV using saccades, which is carried out in a dynamic mode corresponding to one-dimensional scanning of FQV. In general, such 1D scanning is similar to human eye movement $[16,17,18]$, which looks like a series of saccades (super-saccades) and fixations. In this case, the spatial orientation of the local quality as an element of the FQV coincides with the local orientation of the corresponding structural quantum of the assessment of weak 1D videoinformation and allows you to direct the scanning process. In turn, the "weight" of each FQV in their linear combination is determined by the scalar value of the physical quantum (0kvalt) as an element of the FQV.

At the macrolevel of the world outlook, FQVs can be qualitatively compared to strings, which are considered by string theory as fundamental elementary structures of the microworld. If we assume that the string metaphor of the macrocosm is productive, then the next in the hierarchy is the fundamental concept of the microcosm, namely, the 1-brane, most of all corresponds to the semantic content of the global 1qualt, which can be compared to the 1-variety. In this case, the global 1-qualt is a composite dimensional 1D structure with elements that are dynamically formed by super-saccades. Dimensional structures are used to represent the structures of manifolds due to the fact that measuring structural (supraphysical) standards are absent in nature, and therefore can be formed only artificially by means of training (self-learning) ISAM.

\section{INTEGRAL QUASI-HOLOGRAPHIC PRINCIPLE OF EVALUATION OF VIDEO-INFORMATION}

The general formalism of constructing evaluation of videoinformation should consider their reformatting into a structural format, skew-symmetry of the designations of the tensors associated with them and their external derivatives. This allows us to describe this formalism in a unified way in the form of the theory of differential forms, considering those degrees of freedom that are provided by the main theorem of external analysis. Particular cases of this theorem are well known (under the names of Newton-Leibniz, Green, GaussOstrogradsky) and are combined by Stokes' theorem [2, 15, 19, 20]. However, the video- information formalism [4 - 6] was originally formulated in tensor notation. Therefore, mathematically correct, but too abstract and abbreviated designations that are adopted in the theory of differential forms can seriously complicate the construction of information models for evaluation video- information and, moreover, lead the modeling of this process to a dead end. In this regard, tensor designations will be used to construct evaluation of video-information. In this case, the ideas of the mathematical theory of differential forms will be considered, but in a more physicalized form and not so abstractly as is customary in modern mathematics.

Indeed, in the global coordinates of the effective Riemannian space-time, the video information in accordance with (1) has the form.

$t_{n}^{m}=t_{n(E)}^{m}+t_{n(S)}^{m}$
In the case of a nondegenerate metric tensor of the Minkowski space $\gamma_{m n}$ in expression (14), it can be used to lower the contravariant index and write the density of the video information tensor as a sum of symmetric $(s y m)$ and skewsymmetric (alt) components. However, in order to avoid unnecessary cumbersomeness of expressions, the Minkowski space in artificial vision is conveniently considered in rectangular (Euclidean) coordinates. In this case, there is no distinction between superscripts and subscripts, and all indices, for example, can be considered as subscript.

$t_{m n}=t_{m n}^{(s y m)}+t_{m n}^{(a l t)}$

where

$t_{m n}^{(s y m)}=\frac{1}{2}\left(t_{m n}+t_{n m}\right) ; t_{m n}^{(a l t)}=\frac{1}{2}\left(t_{m n}-t_{n m}\right)$

Considering (15), expression (14) can be written in the form

$t_{m n}=t_{m n(E)}+t_{m n(E)}=t_{m n}^{(s y m)}+t_{m n}^{(a l t)}$

where

$t_{m n(E)}=t_{m n}^{(s y m)}$ - physical (symmetric) tensor component of video-information in global coordinates of Minkowski space;

$t_{m n(S)}=t_{m n}^{(a l t)} \quad$ - structural (skew-symmetric) tensor component of video-information in global coordinates of Minkowski space.

In the material macrocosm that surrounds us, the videointelligent technology of artificial vision is initiated by the short-range action of the video-receiver and weak videoinformation through weak video-information field, the source of which is weak video-information. In this case, weak videoinformation is usually considered in terms of intensity.

$$
\begin{aligned}
& {\stackrel{(0)}{I_{m n}}}=\left(\begin{array}{l}
(0) \\
t_{m n}
\end{array}\right)^{2}=\left(\begin{array}{l}
(0) \\
t_{m n(E)}^{(0)}+t_{m n(S)}
\end{array}\right)^{2}= \\
& =\left(\begin{array}{l}
(0) \\
t_{m n}^{(\text {sym })}+t_{m n}^{(\text {alt })}
\end{array}\right)^{2} \rightarrow 2 t_{m n}^{(0)}{ }^{(\text {sym })} t_{m n}^{(\text {alt })}
\end{aligned}
$$

The expression for evaluation the intensity of weak videoinformation based on (17) and (18) can be written in the form.

$$
\begin{aligned}
& \hat{(0)} \hat{I}_{m n}=\left(\begin{array}{l}
(\hat{0}) \\
t_{m n}
\end{array}\right)^{2}=\left(\begin{array}{cc}
\hat{(0)} & \hat{(0)} \\
t_{m n(E)}+t_{m n(E)}
\end{array}\right)^{2}= \\
& =\left(\begin{array}{cc}
\hat{(0)} & (\hat{0}) \\
t_{m n}^{(s y m)} & \left(t_{m n}^{(a l t)}\right.
\end{array}\right)^{2} \rightarrow 2 \hat{t}_{m n}^{(\text {sym })} \hat{t_{m n}^{(a l t)}}
\end{aligned}
$$


It follows from (19) that the estimate of the intensity of weak video- information is determined by the product of its physical (symmetric) and structural (skew-symmetric) tensor components. The video-receiver, after appropriate physical measurements, forms an evaluation of the physical component of weak video-information, which is considered as a physical picture and is sometimes interpreted as an image, which, generally speaking, is erroneous.

In fact, when a person observes a physical picture he evaluates (sees) together the physical and structural components of the evaluation of weak video-information. In this case, the evaluation of the structural (abstract) component of the evaluation of weak video information, which the physical video-receiver is fundamentally unable to register, is formed as a result of the mental activity of the human brain. In other words, when observing a physical picture during several initial stages of vision (natural or artificial), evaluations of the elements of the structural component of weak videoinformation are very quickly synthesized artificially, which correspond to the elements of this physical picture. Further, a joint interpretation of the physically measured and abstractly synthesized elements of these evaluations is carried out, which is accompanied by the construction and recognition of onedimensional and two-dimensional images. As a result, an image as a picture, in which the corresponding physical and structural components of weak video-information are presented together, can only be constructed virtually on the basis of joint physical measurements and structural representations in the mental space of the human brain or in a brain-like artificial vision system.

Let us consider the features of constructing evaluations of the physical (symmetric) and structural (skew-symmetric) components of weak video information. The process of constructing a discrete physical picture by any physical videoreceiver (from a video camera to a multi-element antenna array) is almost always carried out in a similar way. In the era of digital technologies, the most natural for artificial vision is a digital holographic video-receiver based on an incoherent binocular optical video-receiver or a coherent non-optical matrix video-receiver. These physical video-receivers use the optimal video-reception based on a matched filter, which greatly facilitates the analysis of the corresponding holographic process and allows you to completely meaningfully create discrete information models of coherent and incoherent holographic video-receivers adapted to ISAM. For example, in the case of a coherent video- receiver, it is possible to construct a video-informational model [9] on the basis of a weak DHP (2) for evaluating the discrete physical component of weak video-information in the form.

$$
\begin{gathered}
\hat{(0)} \\
t_{m n}^{(s y m)}[r, \Delta z, \Delta \tau]= \\
=\int_{-\infty}^{+\infty} \int_{m n}^{(0)} t_{m n}^{(0) *}(r) t_{m n}^{0(s y m)}\left[r^{\prime}\right] W_{(c \llbracket 0)}(\Delta x, \Delta y \Delta z, \Delta \tau) d x^{\prime} d y^{\prime}= \\
=\int_{-\infty}^{+\infty} \int_{m n}^{(0)} t_{m y)}^{(s y m)}(r) t_{m n}^{(0) *}\left[t^{(s y m)}\left[r^{\prime}\right] W_{(c \square 0)}(\Delta x, \Delta y \Delta z, \Delta \tau) d x^{\prime} d y^{\prime} .\right.
\end{gathered}
$$

Here and below, square brackets are used to symbolically denote discrete functions. Moreover, in expression (20) ${\stackrel{(0)}{t_{m n(E)}}}_{(r)}=\stackrel{(0)}{t_{m n}^{(s y m)}}(r)$ - physical component of weak videoinformation;

$r(x, y, z)$ - coordinates in the area of definition of weak video-information;

$r^{\prime}\left(x^{\prime}, y^{\prime}, z^{\prime}\right)$ - coordinates in the area of definition of the virtual physical component of the reference weak video-information;

$t, t^{\prime}, \Delta \tau, c$ - respectively, the time coordinates of the object (reflected) and reference weak video-information fields, their time shift and propagation speed in the considered physical environment;

$$
\begin{aligned}
& t_{m n}^{(0) *}=\operatorname{comb} b\left(\frac{x^{\prime}}{b}\right) \operatorname{comb}\left(\frac{y^{\prime}}{b}\right)= \\
& =\sum_{m=-\infty}^{m} \sum_{n=-\infty}^{m=+\infty} \delta\left(\frac{x^{\prime}}{b}-m\right) \delta\left(\frac{y^{\prime}}{b}-n\right)=\left\{\begin{array}{l}
1, n p u x^{\prime}=m b, y^{\prime}=n b \\
0, \text { npu } x^{\prime} \neq m b, y^{\prime} \neq n b
\end{array}\right.
\end{aligned}
$$

- the physical component of the weak reference videoinformation is virtually formed by the physical (programmable, "genetic") proto-thesaurus and provides an ideal spatial sampling of weak physical video-information in the form of a two-dimensional lattice $\delta$ - functions;

$$
W_{(c \square 0)}(\Delta x, \Delta y, \Delta z, \Delta \tau)=W_{R_{z}}(\Delta x, \Delta y, \Delta z) W_{z(c \square 0)}(\Delta \tau)-\text { the }
$$
weight function of the ISAM physical video-receiver, which in the quasi-stationary approximation mode has the form of a multidimensional correlation function with factorization into spatial and temporal components;

$$
\begin{aligned}
& W_{R_{z}}(\Delta x, \Delta y, \Delta z)=\int_{-\infty}^{+\infty} \int_{A_{1}}\left(r_{1}\right) \frac{1}{z z^{\prime}} \exp \left\{-j \omega_{0} \frac{R_{z}-R_{z^{\prime}}}{c}\right\} d \mu d v, \\
& W_{z(c \square 0)}=\int_{-\infty}^{+\infty} U\left(t-\frac{2 z}{c}\right) U_{0}^{\prime}\left(t-\frac{2 z^{\prime}}{c}\right) d t
\end{aligned}
$$

- spatial and temporal components of the weighting function of the physical video-receiver, which maintain its selectivity in the transverse and longitudinal directions;

$$
\begin{aligned}
& R_{z} \cong z+\frac{\left(\mu-x_{z}\right)^{2}}{2 z}+\frac{\left(v-y_{z}\right)^{2}}{2 z} ; \\
& R_{z}^{\prime} \cong z^{\prime}+\frac{\left(\mu-x_{z^{\prime}}^{\prime}\right)^{2}}{2 z}+\frac{\left(v-y_{z^{\prime}}\right)^{2}}{2 z} ;
\end{aligned}
$$

$\mu, v$ - coordinates in the opening plane of the physical aperture of the video-receiver;

$$
\hat{t}_{m n}^{(0)}[r, \Delta z, \Delta \tau]-\text { evaluation of the physical component of }
$$
weak video-information in the form of a set of structureless physical samples, the spatial frequency of which is determined by the lattice $\delta$ - functions of the physical proto-thesaurus, and 
their shape coincides with the weight function of the physical component of the video-receiver.

The supra-physical (abstract) nature of the evaluation of the structural component of weak video-information supports the video-information model of the structural interaction of the corresponding supra-physical components, for the implementation of which physical intermediary fields are not needed. Thus, under these conditions, the supra-physical interaction, due to its abstract nature, can (and should) be considered as a long-range action-measurement, the speed of which is infinitely high $(c \rightarrow \infty)$ and this determines the instantaneous nature of such interaction. Under these conditions, the weight function of the structural component of the video-receiver necessarily degenerates into a certain scalar quantity, which, without loss of generality, can be identified with unity. With regard to information models, this means a formal transition from the super-position integral (20) to the "scalar product" (22) of the surface (line, volume, etc.) and the corresponding differential form.

$$
\begin{aligned}
& \text { (0) } \\
& t_{m n}^{(a l t)}\left(r^{\prime}, a\right)= \\
& =d\left\{\int_{-\infty}^{+\infty} \int_{m n}^{(0)} t_{m n}^{(s y m)}(r) t_{m n}^{(0) *}\left[r^{\prime}\right] W_{(c \square 0)}(\Delta x, \Delta y, \Delta z, \Delta \tau) d x^{\prime} d y^{\prime}\right\} \rightarrow
\end{aligned}
$$

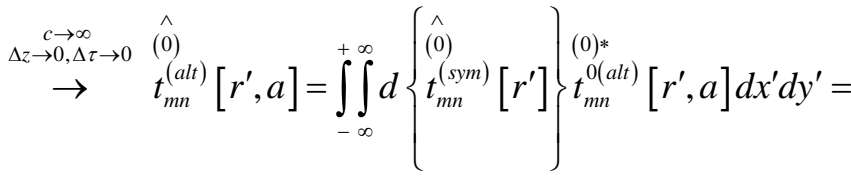

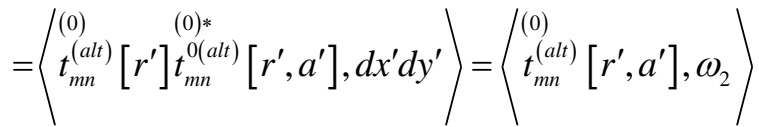

In this expression, $d x^{\prime} \wedge d y^{\prime}=-d y^{\prime} \wedge d x^{\prime}=\omega_{2}$ it is a record in differential form of a basis in the space of skew-symmetric tensors, and the product symbol $\wedge$ is introduced due to the fact that the orientation of the corresponding surface is essential.

Generally speaking, the concepts of tensors and differential forms should be understood in an extended form, i.e. in the form of a field on some manifold $M$, and not at some spatial point of this manifold.

From expression (22) it follows that at the initial stage of video-information restoration in accordance with the inverse problem of artificial vision, the external derivative of the set of physical samples (scalar values) of the physical component of the evaluation of weak video-information is formed. Thus, the structuring of physical samples for evaluation weak videoinformation is carried out by means of their structural reformatting into a set of differential 1-forms. These 1-forms are considered as structural quanta (local qualities) for evaluation weak video-information, which, in contrast to 0forms, have orientations in space. The structural protothesaurus $t_{m n}^{0(\text { alt })}\left[r^{\prime}, a^{\prime}\right]$ determines (measures) these orientations.
Expression (22) also includes the following quantities:

$$
\begin{gathered}
W_{(c \sqsubset 0)}(\Delta x, \Delta y, \Delta z, \Delta \tau) \stackrel{\substack{c \rightarrow \infty \\
\Delta z \rightarrow 0, \Delta \tau \rightarrow 0}}{\rightarrow} W_{(c \rightarrow \infty)}(\Delta x, \Delta y) \rightarrow \\
\rightarrow \text { scalar } \stackrel{\rightarrow \text { without loss of generality }}{\rightarrow} 1
\end{gathered}
$$

- the weight function of the structural component of the video-receiver corresponds to the structural (supra-physical) long-range action and can be taken equal to one;

$$
\begin{aligned}
& t_{m n}^{(0) *}\left[r^{\prime}\right] \rightarrow t_{m n}^{0(\text { alt })}\left[r^{\prime}, a_{i}\right]= \\
& =\left\{\operatorname{comb}\left(\frac{x^{\prime}}{b}\right) \operatorname{comb}\left(\frac{y^{\prime}}{b}\right)\right\} * * \bigcup_{i} \gamma_{\wedge}\left(a_{i}, l_{i}, x^{\prime}, y^{\prime}\right)= \\
& =\left\{\begin{array}{l}
a_{i}, \text { by } x^{\prime}=m b, y^{\prime}=n b, a_{i}=\operatorname{tg}\left(\alpha_{i}\right), i=0,1,2, \ldots, N ; \\
0, \text { by } x^{\prime} \neq m b, y^{\prime}=n b, a_{i} \neq \operatorname{tg}\left(\alpha_{i}\right), i=0,1,2, \ldots, N ;
\end{array}\right.
\end{aligned}
$$

- the structural component of weak reference videoinformation is formed by a structural proto-thesaurus in the form of a lattice of multibeam stars, which can be compared to local goniometric scales and allow evaluation the tilt angles (orientations) of the corresponding structural quanta (local scales) in the video-receiver opening plane;

** - mathematical symbol of two-dimensional convolution;

$$
a_{i}=\operatorname{tg}\left(\alpha_{i}\right)-\text { parameter of the spatial orientation of } i \text { the }
$$
local quality, the length of which $l_{i}=l=$ const, a $\alpha_{i}$ is the angle of inclination (orientation) of the $i$ local quality (1form), locally measured by the structural proto-thesaurus.

Expression (24) assumes the local structure of the protothesaurus in the form of a multi-beam star $\gamma_{\times}(25)$, which is a union $n$ of segments ("rays") with different parameters of spatial orientation $a_{i}$ at the same length $l_{i}=l$ and zero width. The number of rays determines the discreteness of angular measurements $\alpha_{i}$, and all rays intersect at a point (center) with coordinates.

$\gamma_{\times}=\bigcup_{i} \gamma_{\wedge}\left(a_{i}, l_{i}, x^{\prime}, y^{\prime}\right)$

On the whole, the structural proto-thesaurus is programmable and can be considered as a two-dimensional array of multibeam stars, the centers of which coincide with the centers of structureless physical samples. In this case, each multibeam star is functionally a local measuring scale of orientations only for one local samples, and together all these scales make it possible to measure (parallel or parallelsequentially) the spatial orientations of all local qualts and thus, based on (22), reformat physical samples into elementary "mental" video-structures (into structural quanta or, what is the same, into local qualts). 
In the original physical information model (20), the weight function of the physical component of the video-receiver has the form of the kernel of the Fourier-Fresnel transform, which provides the physical component of the video-receiver with the necessary space-time selectivity. As a result of structural reformatting of video-data, the structural component of the video-receiver loses its spatial selectivity, since its weight function becomes a scalar. As you know, a scalar is characterized only by its numerical value (modulus) and has no direction, which allows (without loss of generality and in order to avoid possible confusion) to identify the numerical value of this scalar with unity.

Usually the dot product is used in the traditional way, namely, as a mapping into some quantitative result, which has the form of a real number (ratio). In the application to videointelligent technologies of artificial vision, the concept of scalar product, considering the duality of mathematics, it is advisable to use not in the traditional quantitative form, but in the qualitative form of mapping into a certain dimensional structure (system of relations) as a qualitative (mathematical, structural) process.

Many mathematical concepts, including, for example, a linear combination, have an ambiguity (duality) and therefore can be represented either quantitatively (in the form of numerical values) or qualitatively (in the form of mathematical expressions). In particular, a linear combination can have a quantitative form of an arbitrary number of relations in the form of a set of real numbers that together form a certain quantitative result. However, a linear combination can also have a qualitative form in the form of a system of relations or a structure (a mathematical expression of some process). Unlike a linear combination, a bundle will always have only a qualitative (structural) form and can represent the boundary of a manifold as a union of layers (submanifolds) whose dimension is one less than the dimension of the original manifold. It should be noted that the ambiguity of mathematics excludes the possibility of using a mathematical concept simultaneously in two forms - quantitative and qualitative.

Considering all the circumstances noted, expression (22), which corresponds to the simplest implementation of the integral quasi-holographic principle (IQHP), in a more general form (at some k-th level of the hierarchy) can be rewritten as.

$$
\begin{aligned}
& \begin{array}{c}
\text { Video-data } \\
\text { of dimension } k
\end{array} \\
& \text { in the form of a bundle on } \\
& \text { evaluations of congruent } \\
& \begin{array}{l}
\text { video-information } \\
\text { of dimension } k-1
\end{array} \\
& \text { Video-thesaurus: } \\
& \text { the result of video-data } \\
& \text { accumulation in the form } \\
& \begin{array}{c}
\text { of a } k \text {-scale for } \\
\text { high-quality }
\end{array} \\
& \text { high-quality } \\
& \begin{array}{l}
\text { Self - } \\
\text { study }
\end{array} \\
& \bigcup_{N} \hat{t}_{m n(k-1), n}^{(a l t)}=t_{m n(k)}^{(a l t)} \rightarrow \bigcup_{B} t_{m n(k), \beta}^{(a l t)}=t_{m n(k), \beta}^{0(a l t)} \rightarrow \\
& \begin{array}{l}
\text { "Mental" } \\
\text { evaluation }
\end{array} \\
& \text { evaluation of } \\
& \begin{array}{l}
\text { video-information } \\
\text { of dimension } k
\end{array} \\
& \rightarrow\left\langle\begin{array}{cc}
I Q H P \text { dimension } k \\
t_{m n(k)}^{(\text {alt })} t_{m n(k), \beta}^{0(\text { alt })}, & \omega_{k}
\end{array}\right\rangle \rightarrow \stackrel{\hat{t}_{m n(k)}^{(a l t)}}{\rightarrow}
\end{aligned}
$$

where$$
\hat{t_{m n(k-1)}^{(a l t)}} \text { - evaluation of video-information at the }(k-1) \text {-th }
$$
stage of the ascending hierarchy of artificial vision;

$$
t_{m n(k)}^{0(a l t)}=\bigcup_{B} \hat{t_{m n(k), \beta}^{(a l t)}} \text { - video-thesaurus as a quality measuring }
$$

scale, which is formed by accumulating and combining videodata at the k-th stage of the ascending hierarchy of artificial vision;

$$
\hat{t}_{m n(k)}^{(a l t)} \text { - evaluation of video-information at the k-th stage of }
$$
the ascending hierarchy of artificial vision.

Dynamic linear combinations of FQVs can be considered as 1D dynamic dimensional video-structures that are formed by artificial saccades and allow one to evaluate (measure) 1D video-information together physically (quantitatively) and structurally (qualitatively). An enlarged dynamic linear combination of FQV in the form of a super-saccade can be considered as a composite 1D global dynamic dimensional structure, which can then be used to construct an evaluation of $2 \mathrm{D}$ video-information. In turn, the evaluation of $2 \mathrm{D}$ videoinformation can be considered as a composite dimensional structure for constructing an evaluation of 3D videoinformation, and the evaluation of $3 \mathrm{D}$ video-information, in turn, can be used as a dimensional structure for constructing an evaluation of $4 \mathrm{D}$ video- information.

The hierarchy of integral quasi-holographic videoinformation models of artificial vision can be built on the basis of IQHP, in which physical coherence is not considered due to the absence of physical intermediary fields. However, not only physical fields presented in the energy format of matter, but also supra-physical fields in the structural (abstract) format, can be considered as coherent (comparable) ones. In this case, the geometric congruence (comparability) of video-structures can be considered as a structural analog of physical coherence. Only qualitative measurements of video-information are possible, which in the absence of physical intermediary fields are performed instantly due to the qualitative nature of longrange video-measurements, but only by comparison, since qualitative calculations are not possible.

In accordance with the scheme-formula (26), manifolds of dimension $k$ can be identically represented by bundles (partitions) into submanifolds of boundaries of dimension $k-1$, and this cannot be done by submanifolds of dimension $k-2$.

This means that the only significant dimensional elements that provide adequate sampling of $2 \mathrm{D}$ evaluation of videoinformation are 1D evaluation of video-information. According to the classical axiom of Archimedes, "any segment can be measured using any other" [21]. Therefore, for each of these 1D layers, the super-saccades in the form of enlarged artificial saccades will be the elementary dimensional structures. They can combine partial FQVs by scanning either in accordance with their "internal" spatial orientation of FQVs (during 
training / self-learning), or on the basis of "external" spatial orientation, which in ISAM artificial vision is dictated by hierarchically descending video-informational feedback.

Accordingly, in the 3D case, the only significant sampling elements will be only 2D evaluation of video-information (in the form of images mapped to surfaces). In this case, each 2D evaluation of video- information will be compared, generally speaking, to the corresponding observed 2D area of the surface of the controlled 3D object. Finally, in the 4D case, the threedimensional images of geometric bodies formed in the process of learning (self-learning) in the form of 3D evaluation of video- information and the 3-manifolds associated with them will be considered as the only significant "dimensional" elements.

The schematic formula (26) can be associated with a certain $k$ stage of the ascending hierarchy, which implements a quasiholographic abstract long-range action-measurement and generates an evaluation of a video-information of dimension $k$ . In general, the structural and functional architecture of the ISAM video-component can be represented by a formal scheme (27), which, in turn, can be compared to the structure of the visual area of the artificial neocortex as the intellectual core of ISAM.

$$
\begin{aligned}
& \begin{array}{ccc}
\text { Full video-data } & \begin{array}{c}
\text { 1D dynamic video-data } \\
\text { (super-saccades): }
\end{array} & \begin{array}{c}
\text { 1D video-thesaurus: accumula- } \\
\text { tion of 1D dynamic video-data }
\end{array}
\end{array} \\
& \begin{array}{lll}
\text { Fulvideo-data } & \text { (super-saccades): } & \text { tion of } 1 \mathrm{D} \text { dynamic video-data } \\
\text { quanta }(\mathrm{FVQ}) & \text { combining artificial saccades } & \text { 1D } \\
\text { (super-saccades) in the form }
\end{array} \\
& \begin{array}{ccc}
\begin{array}{c}
\text { combining artificial saccades } \\
\text { and controlling their }
\end{array} & \text { 1D } & \text { (super-saccades) in the form } \\
\text { scanning } & \text { self- of a 1D scale for high-quality } \\
\text { study } & \text { measurements }
\end{array} \\
& t_{m n(0)}^{(s u m)} t_{m n(0)}^{(a l t)}=t_{m n(0)} \rightarrow \bigcup_{I} t_{m n(0), i}=t_{m n(1)} \rightarrow \bigcup_{Q} t_{m n(1), q} \rightarrow \\
& \begin{array}{ccc}
\text { 2D video-data } \\
\text { 1D IQHP } & \begin{array}{c}
\text { Mental" evaluation } \\
\text { of 1D weak } \\
\text { video-information }
\end{array} & \begin{array}{c}
\text { in the form of a bundle } \\
\text { 1D congent evaluation } \\
\text { 1D video-informations }
\end{array}
\end{array} \\
& \rightarrow\left\langle t_{m n(1)} \bigcup_{Q} t_{m n(1), q}, \omega_{1}\right\rangle \rightarrow \hat{t_{m n(1)}^{(a l t)}} \rightarrow \bigcup_{L} \hat{t}_{m n(1), l}^{(a l t)}=t_{m n(2)}^{(a l t)} \rightarrow \\
& 2 \mathrm{D} \text { video-thesaurus: } \\
& 2 D \begin{array}{c}
\text { accumulation of 2D video-data } \\
\text { in the form of a 2D scale }
\end{array} \\
& \begin{array}{l}
\text { self- } \\
\text { study }
\end{array} \\
& \rightarrow \quad \bigcup_{L} t_{m n(2), l}^{(a l t)} \rightarrow\left\langle t_{m n(2)}^{(a l t)} \bigcup_{L} t_{m n(2), l}^{(a l t)}, \omega_{2}\right\rangle \rightarrow t_{m n(2)}^{(a l t)} \rightarrow \\
& \begin{array}{ll}
\begin{array}{c}
\text { 3D video-data } \\
\text { in the form of a bundleo-thesaurus: } \\
\text { of congruent evaluation }
\end{array} & \begin{array}{c}
\text { 3D } \\
\text { accumulation of 3D video-data } \\
\text { in the form of a 3D scale }
\end{array}
\end{array} \\
& 1 \mathrm{D} \text { video-informations self- for high-quality measurements } \\
& \rightarrow \bigcup_{K} t_{m n(2), k}^{(a l t)}=t_{m n(3)}^{(a l t)} \rightarrow \bigcup_{K} t_{m n(3), k}^{(a l t)} \rightarrow \\
& \begin{array}{ccc}
\text { 4D video-data } & \begin{array}{c}
\text { Mental" evaluation } \\
\text { of 3D } \\
\text { video-information }
\end{array} & \begin{array}{c}
\text { in the form of a bundle } \\
\text { of congruent evaluation } \\
\text { 1D video-informations }
\end{array}
\end{array} \\
& \rightarrow\left\langle t_{m n(3)}^{(a l t)} \bigcup_{K} t_{m n(3), k}^{(\text {alt })}, \omega_{3}\right\rangle \rightarrow t_{m n(3)}^{(\text {alt })} \rightarrow \bigcup_{M} t_{m n(3), m}^{(\text {alt })}=t_{m n(4)}^{(\text {alt })} \rightarrow
\end{aligned}
$$

Video-intelligence: accumulation of $4 \mathrm{D}$ video-data in the form of a $4 \mathrm{D}$ scale for high-quality measurements "Mental" evaluation of 4D

$$
\rightarrow \bigcup_{M} t_{m n(4), m}^{(a l t)} \rightarrow\left\langle t_{m n(4)}^{(a l t)} \bigcup_{M} t_{m n(4), m}^{(a l t)}, \omega_{4}\right\rangle \rightarrow t_{m n(4)}^{(a l t) \stackrel{(27)}{\wedge} \text { (2ideo-information }}
$$

Here $\quad t_{m n(0)}^{(s u m)} t_{m n(0)}^{(\text {alt })}=t_{m n(0)} \quad-$ tensor field $\quad \mathrm{FVQ}$; $\bigcup_{I} t_{m n(0), i}=t_{m n(1)}-$ enlarged artificial saccades (supersaccades), which are considered as 1D "dimensional" elements of the global evaluation of 1D video-information;

$$
\bigcup_{Q} t_{m n(1), q}-1 \mathrm{D} \text { video-thesaurus as a combination of }
$$
super-saccades into dimensional 1D video-data, which form a quality measuring scale for evaluation 1D weak videoinformation;

$$
\left\langle t_{m n(1)} \bigcup_{Q} t_{m n(1), q}, \omega_{1}\right\rangle-1 \mathrm{D} \text { IQHP, which generates a }
$$
"mental" $1 \mathrm{D}$ evaluation of weak video- information in the form of splitting into congruent super-saccades $t_{m n(1)}^{(a l t)}$;

$$
\bigcup_{L} t_{m n(1), l}^{(a l t)}=t_{m n(2)}^{(a l t)} \quad-2 \mathrm{D} \text { video-data in the form of a bundle }
$$
of congruent $1 \mathrm{D}$ evaluations video-information;

$$
\bigcup_{L} t_{m n(2), l}^{(a l t)}-2 \mathrm{D} \text { video-thesaurus in the process of self- }
$$
learning combines 2D video-data into 2D high-quality measuring scale for evaluation (measuring) 2D weak videoinformation;

$$
\left\langle t_{m n(2)}^{(a l t)} \bigcup_{L} t_{m n(2), l}^{(a l t)}, \omega_{2}\right\rangle-2 \mathrm{D} \text { IQHP, which generates, as a }
$$
result of long-range interaction-measurement a "mental" $2 \mathrm{D}$ evaluation of weak video-information $\hat{t}_{m n(2)}^{(a l t)}$.

$$
\bigcup_{K} t_{m n(2), k}^{(a l t)}=t_{m n(3)}^{(a l t)}-3 \mathrm{D} \text { video-data in the form of a bundle }
$$
of congruent 2D video-information evaluations;

$$
\bigcup_{K} t_{m n(3), k}^{(a l t)}-3 \mathrm{D} \text { video-thesaurus combines 3D video-data }
$$
through self-learning and thereby forms a high-quality 3D measurement scale; 
$\left\langle t_{m n(3)}^{(a l t)} \bigcup_{K} t_{m n(3), k}^{(a l t)}, \omega_{3}\right\rangle-3 \mathrm{D}$ IQHP, which generates a

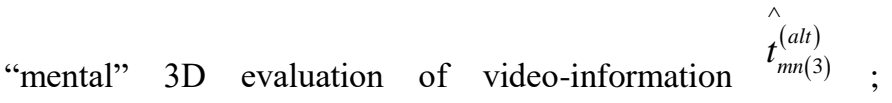
$\bigcup_{M} t_{m n(3), m}^{(a l t)}=t_{m n(4)}^{(a l t)}$ congruent $3 \mathrm{D}$ video-information evaluations;

$$
\bigcup_{M} t_{m n(4), m}^{(a l t)}
$$

- 4D video-thesaurus combines (by selflearning) 4D video-data and forming a quality measurement 4D scale;

$$
\left\langle t_{m n(4)}^{(a l t)} \bigcup_{M} t_{m n(4), m}^{(a l t)}, \omega_{4}\right\rangle
$$

- 4D IQHP that generates "mental"

4D evaluation of video-information $\hat{t}_{m n(4)}^{(a l t)}$.

It should be noted that in the constructed model of the ascending hierarchy of video-information evaluations, the previously proposed approach [8] based on video-information layering and the approach based on IQHP are interconnected, used jointly and complement each other within the framework of the intelligent technology of the ISAM video-component. The result is a unified video-intelligent mechanism of the ascending hierarchy of video-information quality evaluations.

\section{FULL SAMPLING THEOREM FOR VIDEO-INFORMATION}

The outer derivative of a differential form is a natural generalization of the well-known gradient, rotor, and divergence operations. Therefore, the general definition of integrals (and iterated integrals) of differential forms [15] allows us to use the Stokes formula. It is more convenient (although not entirely customary) to represent this formula in pairing notation $[23,24]$ or, which is the same, in terms of scalar products. In the case of $k$ - dimensional manifolds $(k=\overline{1,4})$, this version of the Stokes formula in global rectangular coordinates of the Minkowski space can be written as.

$$
\left\langle\partial \hat{t}_{m n(k)}^{(a l t)}, \omega_{k-1}\right\rangle=\left\langle\hat{t}_{m n(k)}^{(a l t)}, d \omega_{k-1}\right\rangle
$$

where

$$
\hat{t_{m n(k)}^{(a l t)}}-\text { evaluation of video-information associated with } k \text { - }
$$

manifold;

$$
\partial \hat{t}_{m n(k)}^{(a l t)}=t_{m n(k-1)}^{(a l t)}-\text { the boundary of a } k \text {-dimensional }
$$
manifold in the form $(k-1)$ of submanifolds forming a bundle; $\omega_{k}=d x_{1}^{\prime} \wedge \ldots \wedge d x_{k}^{\prime}$ - basis of the space of skewsymmetric tensors in differential form of dimension $k$;

$$
d \omega_{k-1}=\omega_{k}-\text { the outer differential of the basis of the space }
$$
of skew-symmetric tensors in the differential form of dimension $k$.

It is known that in the formulation of the general Stokes theorem it is not necessary to assume that the boundary of the manifold consists entirely of one piece. The boundary of a manifold can be a set of pieces of the same dimension. For example, a boundary can have the form of dynamic linear combinations of FQVs (in the form of saccades and supersaccades) or the form of bundles (partitions) into submanifolds of the same dimension (by one less than the dimension of the original manifold), called layers.

Considering the noted circumstances, expression (28) can be rewritten as

$$
\begin{aligned}
& \text { "Mental" } \quad \text { Formula for a complete } \\
& \text { evaluation (quantitative and qualitative } \\
& \text { of video-information } \quad \begin{array}{c}
\text { together) sample } \\
\text { of video-information }
\end{array} \\
& \text { of dimension k-1 } \quad \begin{array}{c}
\text { of video-inform } \\
\text { evaluation }
\end{array} \\
& \hat{t}_{m n(k-1)}^{(a l t)}=\partial t_{m n(k)}^{(a l t)} \rightarrow\left\langle\partial t_{m n(k)}^{(a l t)}, \omega_{k-1}\right\rangle= \\
& \text { "Mental" } \\
& \text { of video-information } \\
& \text { of dimension } \mathrm{k} \\
& =\left\langle t_{m n(k)}^{(a l t)}, d \omega_{k-1}\right\rangle \rightarrow \hat{t_{m n(k)}^{(a l t)}}
\end{aligned}
$$

In general, the formal scheme (29) you can match to the new full sampling theorem for assessing video information in the "mental" space of the ISAM, which can be regarded as a further development of the classical Kotelnikov - Shannon sampling theorem in relation to the conditions of full video data, which are considered quantitatively and qualitatively together. The meaning of identity (29) is that a complete sample of video-information of dimension $k$ is divided into arbitrarily small parts only by a set of dimensional evaluation of video-information of dimension $k-1$ and this cannot be done by a set of evaluation of video-information of dimension $k-2$.

Thus, by virtue of (29), the evaluation of the full videoinformation of the dimension $k$ is identical to the partition ("discretization") of this evaluation in the form of a set of dimensional video-information (complete samples) of the dimension $k$-1. Due to the topological nature of these quantities, they can be compared only qualitatively, but not quantitatively. The set of samples obtained in the process of full "sampling" at any level of the ISAM hierarchy may seem excessively large and dense. Apparently, redundancy is a 
characteristic feature of complete (quantitative and qualitative together) measurements. This is the fundamental difference between complete information evaluations and quantitative information evaluations, which support, for example, the construction of a physical picture of an image as an evaluation of the physical component of weak video-information. If in systems for quantitative information evaluation, numerical calculations are permissible in order to increase noise immunity and evaluation accuracy, then in systems for full information evaluation such calculations are impossible and almost the only way to ensure noise immunity is to support the redundancy of a full information evaluation.

\section{HiERARCHICAL FEEDBACK AND STRUCTURAL AND FUNCTIONAL SCHEME OF THE BRAIN-LIKE VIDEO-COMPONENT OF ARTIFICIAL MIND}

Physical (quantitative) feedback between the output and input of a certain physical data processing system can manifest itself, depending on the operating conditions, either as negative feedback, or as partially or completely positive feedback. In artificial vision, a new type of feedback takes place - supraphysical (qualitative) feedback. This feedback is characterized by completely different tasks that are fundamentally different from the tasks of physical feedback in modern cybernetic tools and control systems. Top-down hierarchical feedback provides an instant comparison of the "exit" and "input" states of artificial vision, indicating those 1D directions along which the physical image will be scanned during its structural reformatting by artificial saccades and super-saccades. This is a new (qualitative) type of instantaneous interaction between the output and input of an intelligent system, in which this superphysical long-range action is realized with the help of a descending hierarchical feedback.

The main identity of the formal scheme (29) can be used not only when constructing an ascending (bottom to top) hierarchy for synthesizing video-information evaluations in the form of representation (30) as a direct version of the complete information sampling theorem. Representation (31) as an inverse version of the full information sampling theorem can be used in the feedback for the descending hierarchical service of video-information evaluations.

Due to the dominance of the structural (qualitative) component in the ascending hierarchy of the synthesis of video-information evaluations, the descending service hierarchy in the feedback should have the same structural (qualitative) nature. In the case under consideration, the feedback cannot be negative or positive, but must be of a qualitative, non-computational nature, since qualitative calculations are impossible, and only comparisons and ordering of the attributes of qualitative variables are permissible. The high-quality interaction of the input video data with the output of the downward feedback of artificial vision provides the possibility of high-quality control over the scanning of informative fragments of the input video-data. This is done instantly due to the supra-physical singularity of both the ascending hierarchy of video-information evaluation and the descending hierarchy of video-information feedback.

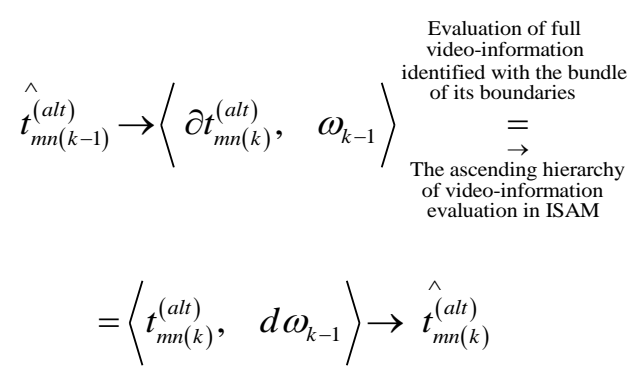

$$
\begin{aligned}
& \text { Boundary bundles } \\
& \begin{array}{l}
\text { identified in the form } \\
\text { of full video- information }
\end{array} \\
& \hat{t}_{m n(k-1)}^{(a l t)} \leftarrow\left\langle\partial t_{m n(k)}^{(a l t)}, \quad \omega_{k-1}\right\rangle \underset{\begin{array}{c}
\text { Downward hierarchy } \\
\text { of service video-information } \\
\text { in the ISAM feedback }
\end{array}}{=} \\
& =\left\langle t_{m n(k)}^{(a l t)}, \quad d \omega_{k-1}\right\rangle \leftarrow \hat{t}_{m n(k)}^{(a l t)}
\end{aligned}
$$

In expression (30), corresponding to the theorem of the full video- information sampling, the mathematical symbol of identity (equality) shows that the fulfillment of identity (30) from left to right allows us to interpret (30) as a formula for of a full video-information sampling using the appropriate bundles (partitions). In the inverse case (31), the direction of the identity from right to left corresponds to the complete (quantitative and qualitative jointly) "discretization" in the descending hierarchy of the feedback overhead. Feedback is formally multivalued, which gives rise to the need to solve the problem of artificially providing unambiguous feedback. To solve this problem, at each level of the hierarchy of the downward feedback, a "mechanism" for ensuring unambiguity is synthesized, which allows the feedback to adequately perform its functions on the formation of actual scanning trajectories of the input video-data (in the form of supersaccades) in real time.

Based on the foregoing, it can be built structurally functional architecture of video-component ISAM, which was surprisingly coincides exactly with the known structure of the human neocortex (the number of rising levels of the hierarchy, the presence of a hierarchy in a direct and feedback, the method of collecting basic video-data, etc.). Due to this exact coincidence, the singularity video- component of the ISAM can be considered as the video-component of the artificial neocortex in the form of intelligent multi-core of the artificial mind (Fig. 1).

ISAM form the full evaluation of video-information in its own virtual ("mental") space as hierarchically ascending evaluations of video-information with coarse (1D, 2D) and finer (3D, 4D) topology. This "mental" space is viewed as a mathematical product of two "mental" subspaces. One of which has the form of a general Riemannian subspace with a local coordinate system and a complex topology (map atlas), and the other represent a Minkowski space with a global coordinate system and with a simple topology given in one map. 
ISAM "mental" space for representing evaluations of video-information in the process of their intellectual recognition, perception and understanding

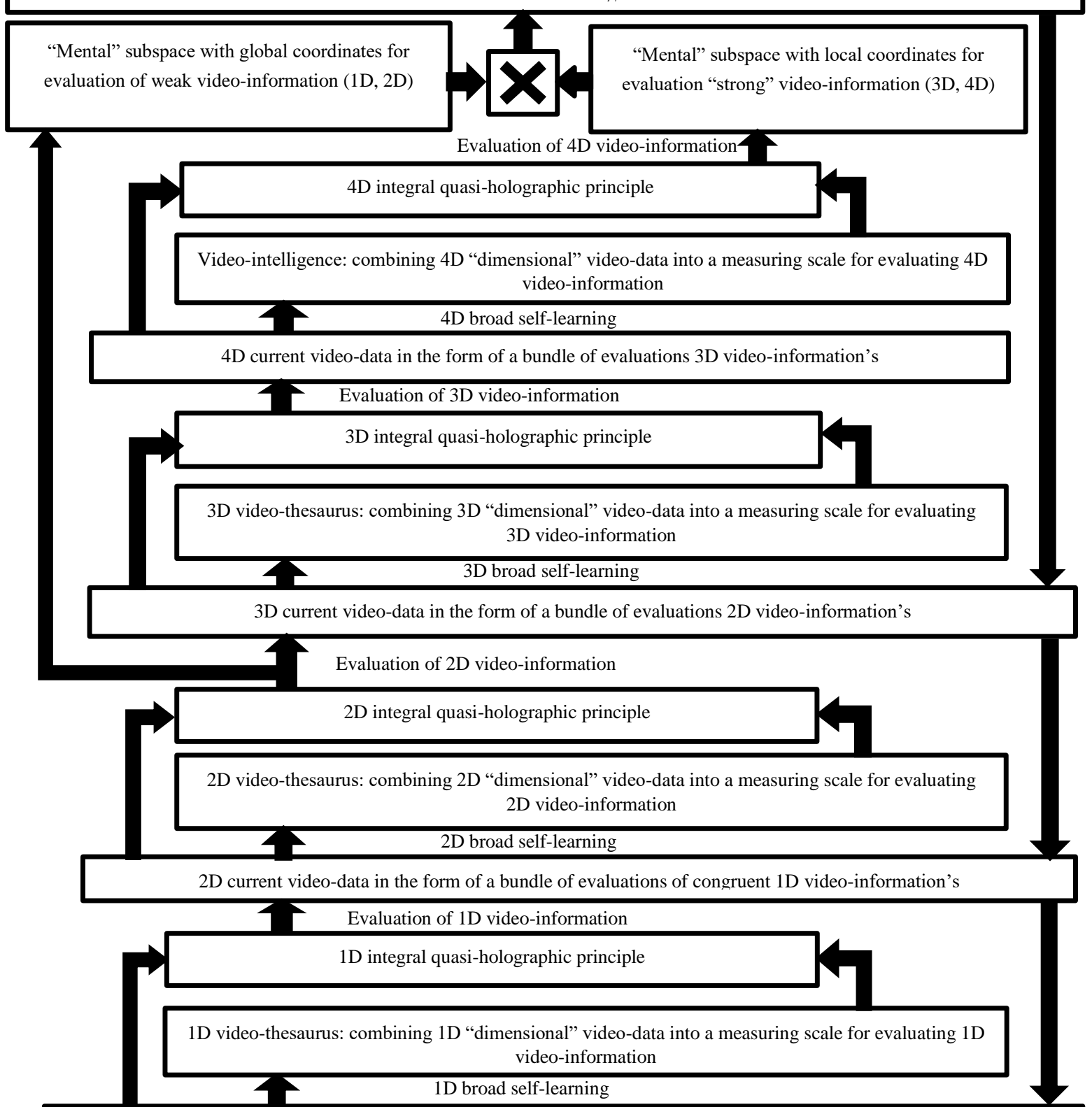

1D "dimensional" dynamic video-data (artificial super-saccades): the direction and density of scanning is set by the 1D output of the ISAM downward feedback

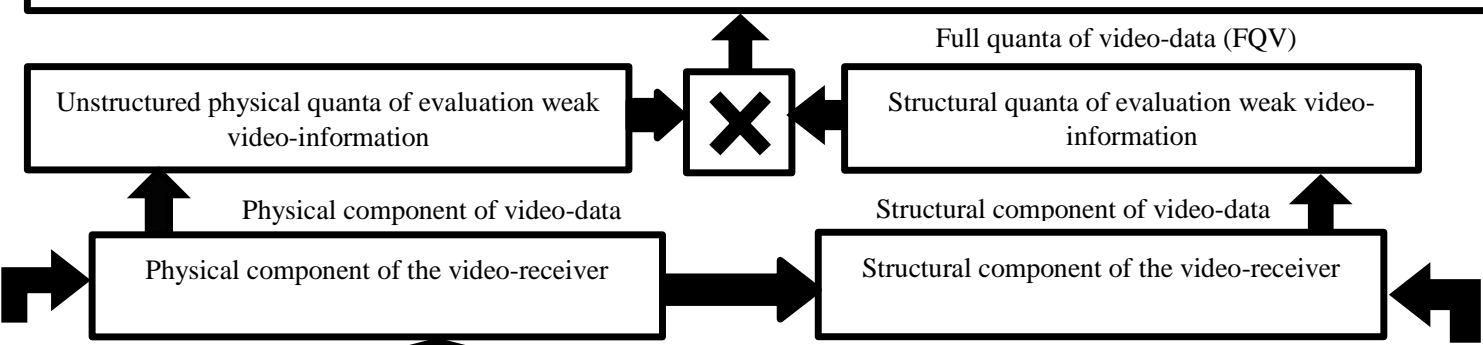

Physical proto-thesaurus of the video-receiver

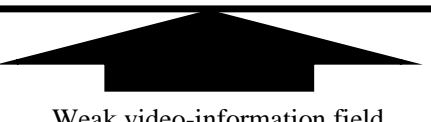

Weak video-information field
Video-receiver structural proto-thesaurus

Fig. 1. Structural and Functional Architecture of the Brain-like Video-component of Artificial Mind. 
The "mental" Riemannian subspace of general view is a curved space. In this subspace, there is a wide scope for constructing abstract formal-mathematical reasoning in local coordinates and actions, for which are not required intuitive clarity. It is enough just formal compliance with the laws of mathematics. In general, "mental" Riemannian subspace can be compared to the mental space of the left hemisphere of the human brain [22], which is mainly responsible for abstract thinking.

In turn, in a flat "mental" subspace, such formalmathematical reasoning and constructions are possible, which are characterized by visualization and intuitive clarity. In this regard, the flat "mental" subspace in ISAM can be compared to the mental space of the right hemisphere of the human brain [22], which is mainly responsible for the direct perception of the surrounding World.

\section{DISCUSSION}

An additional "bonus" to the new (post-Shannon) informational approach is the mathematically accurate and physically correct DHP (strong and weak), which can be considered as a holographic interpretation of this new informational approach.

Structural reformatting (structuring) of physical video-data is carried out in ISAM on the basis of full quanta of video-data (FQV) and artificial saccades, the purpose of which, apparently, is similar to the purpose of saccades of natural human vision.

The effectiveness of video-information processes at all levels of the ISAM hierarchy is ensured by its phased hierarchical training/self-training.

DHP differs from IQHP in that the latter is supra-physical (abstract) and, therefore, singularity (instantaneous).

Generally speaking, ISAM is a kind of brain-like technology of artificial mind in a small (in the form of strong artificial intelligence) with an intelligent multi-core in the form of an artificial neocortex, which is considered as an emulation of the human neocortex.

In ISAM, a general "mental" Riemannian subspace with a local coordinate system can be matched with the mental space of the left hemisphere of the human brain, which is mainly responsible for abstract thinking. In turn, the "mental" subspace of the ISAM with a global coordinate system can be considered as flat or of constant curvature. Therefore, in such a subspace, such formal-mathematical reasoning and constructions are possible, which are characterized intuitive clarity. In this regard, this "mental" subspace of ISAM can be compared to the mental space of the right hemisphere of the human brain, which is mainly responsible for the directly visual perception of the surrounding World. On the whole, the effective Riemannian "mental" space ISAM as a product of partial subspaces-factors has a simple topology given in one chart.

The theorem of supra-physical (qualitative) sampling of video- information evaluations in the "mental" space of ISAM can be considered as a further development of the Kotelnikov-
Shannon sampling theorem in relation to the conditions for evaluating video- information as a qualitative supra-physical quantity. In this case, the set of quality video-sampling may seem excessively large and/or too dense in the information sense. However, this is a characteristic feature of the qualitative evaluation of video-information, which supports the well-known high noise immunity of vision-systems (natural and artificial).

The brain-like nature of artificial mind does not imply full compliance with the anatomical structure of the human brain. The only component of the human brain in which technology is currently showing deep interest is the neocortex [25] or, in other words, the new cortex of the human brain. This homogeneous neuroenvironment with a small number of hierarchical levels supports human thinking and can be emulated by an artificial neocortex, which in turn can be meaningfully built on the basis of post-Shannonian information approach (developed to the level of informational synthesis) and computer methodology.

The video-component of the ISAM is built on the basis of IQHP, is characterized by a singularity (instantaneousness) of video-information evaluation and provides in real time the effectiveness of joint recognition, perception and understanding of the surrounding World when using an artificial neocortex as an intelligent multi-core of artificial mind. It should be noted that the informational methodology of ISAM has no parallels with the heuristic methodology of neural networks and, first of all, in the field of intelligence formation based on learning / self-learning processes, which are "broad" [8], in contrast to the "deep" learning processes adopted in neural networks.

The singularity of the brain-like technology of the videointelligent component of the artificial neocortex as an intelligent multicore of the ISAM supports the instantaneousness of the ascending hierarchy of videoinformation evaluations, which are not only abstract, but also non-linear. Because of this, the artificial neocortex lacks a directly visual internal "mental" World, which is considered as an abstract environment of hierarchically organized internal "dimensional" structures (video structures) generated by the nonlinear intellectual mechanism of the artificial neocortex. "Restructuring" of this intellectual mechanism, for example, in order to achieve greater visibility of recognition, perception and understanding of video-information evaluations, means a transition to other principles of information processing and the loss of the instantaneous nature of this intellectual mechanism.

The intelligent mechanism of artificial mind based on the hierarchy of IQHP, generally speaking, supports the creation of both sensory and supra-sensory intellectual mechanisms of artificial mind. At the same time, for the formation of the corresponding supra-sensory thesauri and intellects (in the field of mathematics, physics, chemistry, computer science and other fundamental and applied sciences), teaching methods can be used in the form of an ascending hierarchy, i.e. from simple facts, phenomena, actions and processes to their increasingly complex presentation.

The fundamental feature of any information is that the instantaneousness of its processing (evaluation) is ensured by 
appropriate structuring, which allows for the joint processing of quantitative (physical) and qualitative (structural) components of information. If you do not take any special measures to structure the training information, then the singularity intellectual mechanism of artificial mind (based on the IQHP) becomes unworkable and the instantaneous assessment of information is not realized. It is possible that such processes inhibiting natural speed of response took place during the development of the natural mind of a person in the process of learning only the quantitative component of knowledge. At the same time, the qualitative (structural) component of knowledge was ignored on the assumption that quantity, in accordance with the well-known law of philosophy, would naturally pass into a new quality. As a result, the mind of a person trained in this way has lost access to his inner, instantly acting intellectual mechanism, which is built on the basis of supra-physical (abstract, qualitative) longrange measurements. To solve the problem of the speed of human civilization that arose, it was necessary to invent abacus, adding machines, calculators and, finally, computers and supercomputers.

At present, there is no hope for the natural appearance of people with superintelligence, and therefore the developer of the artificial superintelligence will not be nature, but that part of the community of people-developers who have realized the "systemic error" of the development of the natural human mind, and will not allow a repetition of this error during artificial formation and the development of machine supermind. The essence of such development is learning / selflearning based on qualitative principles and approaches, which are "inconvenient" in the traditional development of the natural human mind based on quantitative principles and approaches, but much more effective. However, this is a subject for separate consideration.

The artificial neocortex as an intelligent multicore of artificial mind can support numerous supra-sensory intelligent technologies, which are actually technologies of artificial "psyche" and "emotions" [26]. These technologies, considered together, can be compared to artificial consciousness. At the same time, the artificial mind as a material carrier of artificial consciousness is considered as some highly co-intellectual essence of a certain "mental" and "emotional" type.

In response to two types of influences (external and internal), the artificial mind during its development should be able to change the structure and functions of its hardware material carrier (by analogy with the neuroplasticity of the human brain), which is most naturally realized on the basis of broad learning / self-learning. The development of artificial mind presupposes the possibility of significant changes in the patterns of its intellectual activity, as well as the structure and functions of its hardware material carrier.

It is necessary that such internal cardinal restructuring of the artificial mind in the process of its entire development be accompanied by certain efforts of the artificial "psychic" qualities, habits and "emotional" reactions. These new patterns of thinking and behavior will exclude the possibility of even accidental synthesis of negative template of actions in relation to man and human civilization.
The development of artificial mind is unlikely to be possible to regulate certain frameworks, and it will go towards the widest possible coverage of all the latest intelligent technologies that will be considered together. Only in the case of "psychic" and "emotional" positivity of artificial mind as a super-intelligent artificial entity will it be possible to "negotiate" with artificial mind about using its intellectual capabilities to exclude technological singularity and the collapse of human civilization as a whole.

In accordance with the new information approach to artificial intelligence, the artificial neocortex can be viewed as a stratification into numerous sensory and supra-sensory intellectual components. In the case of a limited number of supra-sensory intellectual components, the artificial neocortex acquires the quality of an intelligent multi-core of strong artificial intelligence (artificial mind in the small). The expansion of the number of supra-sensory intellectual components of the artificial neocortex to all types of modern and perspective intellectual activity equips the artificial neocortex with many new intellectual components. This significantly enhances the intellectual capabilities of the artificial neocortex, which, with this development, can be considered as an intellectual multicore of an artificial supermind.

\section{CONCLUSIONS}

1) The differential holographic principle (weak and strong) can be considered as a holographic interpretation of the new (post-Shannon) information approach in the small and in the whole.

2) Structural reformatting of dynamic physical video-data is carried out in ISAM on the basis of full quanta of video-data (FQV) and artificial saccades, which are functionally similar to saccades of natural human vision.

3) The effectiveness of the video-information process at all levels of the ISAM hierarchy is ensured by step-by-step hierarchical training / self-training.

4) IQHP in ISAM is used in the ascending hierarchy of supra-physical long-range measurements and, due to its singularity, allows to instantly solve artificial vision problems (which do not require additional training/self-training) based on instant representation of video-information evaluations in the "mental" space of ISAM.

5) IQHP can be considered as a further development of the holographic idea, within the framework of which the actually existing physical environment of material (physical) close-range action-measurements is replaced by of "mental" (supra-physical) a virtual environment with instantaneous long-range action-measurements in ISAM.

6) ISAM can be built on the basis of a brain-like technology with an intelligent multicore in the form of an artificial neocortex with its own "mental" effective Riemannian space. In this case, the "mental" curved Riemannian subspace of a general form with a local coordinate system can mapped with the mental space of the left hemisphere of the human brain, which is mainly responsible for abstract thinking. In turn, the "mental" sub- 
space with the global coordinate system can be mapped with the mental space of the right hemisphere of the human brain, which is mainly responsible for the direct perception of the surrounding World.

7) The new theorem of full sampling of video information assessment in the ISAM "mental" space can be considered as a further development of the classical Kotelnikov - Shannon sampling theorem in relation to the conditions of singular synthesis of assessments of complete (quantitative and qualitative jointly) video-information's.

8) The intelligent multi-core ISAM in the form of an artificial neocortex emulates the human neocortex and carries out instant "mental" processing (evaluation) of information (video information), which allows us to consider the ISAM as an intelligent singular system. The singularity of this system is an important technological result of an intelligent approachsynthesis of video- information evaluations based on "mental" long-range measurements. This synthesis approach is built on the basis of a bottom-up IQHP hierarchy, broad learning / selflearning, top-down global feedback and computational methodology, but without the traditional intuitive and heuristic ideas of neural networks and deep learning.

\section{REFERENCES}

[1] Yarichin E.M.: Informational Mechanism of Intellectual Technical Vision. IEEE International Siberian Conference on Control and Communications (SIBCON-2011). IEEE Xplore, Digital Library.

[2] Penrose R. The path to reality, or the laws governing the Universe. Complete guide. Translation from English - Izhevsk, Center "Regular and Chaotic Dynamics", (2007), - 911 p. (in Russian).

[3] Greene B. Latent reality: Parallel worlds and deep laws of the cosmos. Translation from English - M.: URSS, Book House "LIBROKOM", (2013), - 400 p. (in Russian).

[4] Yarichin E.M.: Informational Paradigm of Technical Vision. Pattern Recognition and Image Analysis, (2008), Vol. 18, No. 1.

[5] Yarichin E.M.: Theory of full Information (Video-information). IEEE International Siberian Conference on Control and Communications (SIBCON-2011). IEEE Xplore, Digital Library.

[6] Yarichin E.M.: Theory of Full (Video) Information: Bridging of the Gap between Quantity and Quality in Modern Paradigm of Information. International Journal of Information Science, (2013), Vol. 3, №3, p. 3758.

[7] Yarichin E.M.: Video-information: Internal Structure and the Features of the Discretization. International Journal of Engineering and Innovative Technology (IJEIT), November (2013), Vol. 3, № 5, p.133-140.
[8] Yarichin E.M., Gruznov W.M., Yarichina G.F.: Intellectual Paradigm of Artificial Vision: From Video-Intelligence to Strong Artificial Intelligence. (IJACSA) International Journal of Advanced Computer Science and Application, Vol. 9, № 11, (2018), p. 16 - 32.

[9] Kachanov E.I., Pigulevsky E.D., Yarichin E.M.: Methods and means of hydroacoustic holography - L.: Sudostroenie, (1989). - 256 p. (in Russian).

[10] Soroko L.M.: Fundamentals of holography and coherent optics. - M.: Science, Ch. ed. physical-mat. lit., (1971). - 616 p. (in Russian).

[11] Yau S-T., Nadis S. String Theory and Hidden Dimensions of the Universe Translation from English. - SPb.: Peter, (2012). - 400 p. (in Russian).

[12] Randall L. Swirling Passages: Penetrating the Mysteries of the Hidden Dimensions of Space. Translation from English. - M.: URSS, Book House "LIBROKOM", (2011) - 400 p. (in Russian).

[13] Zwieback B. An introductory course in string theory. Translation from English. - M.: URSS, (2013). - 784 p. (in Russian).

[14] Introduction to topology: Textbook for universities / Borisovich Yu.G., Bliznyakov N.M., Izrailevich Ya.A., Fomenko T.N. - M.: Lenand, (2015). - 448 p. (in Russian).

[15] Dubrovin B.A., Novikov S.P., Fomenko A.T.: Modern geometry: Methods and applications -- T1-3. Ed. 6, URSS, (2013) - 920 p. (in Russian).

[16] Haken G., Haken-Krell M. Secrets of perception. Translation from English - Moscow: Institute for Computer Research, 2002 - 272 p. (in Russian)

[17] Zavalishin N.B., Muchnik I.B.: Models of visual perception and algorithms for image analysis. - M., (1974). - 344 p. (in Russian).

[18] Schiffman H.R. Feeling and perception. 5th edition. Translation from English. - SPb.: Peter, (2003). - 928 p. (in Russian).

[19] Ryder L. Quantum field theory. Translation from English. - M.: Mir, (1987), - 511 p. (in Russian).

[20] Deschamps G.A.: Electromagnetic and differential forms, Proc. of the IEEE, (1981), vol. 69, № 6, p. 676 - 696.

[21] Hilbert L., Cohn-Vossen S. Visual geometry. Translated from German. 3rd ed. - M.: Science, (1981). - 344 p. (in Russian).

[22] Sergeev B.F.: Phenomenon of functional asymmetry of the brain. M.: Book House "LIBROKOM", (2014). - 176 p. (in Russian).

[23] Kaku M.: Introduction to superstring theory: Translated from English. M.: Mir, (1999). - 624 p. (in Russian).

[24] Zorich V.A.: The modern Newton-Leibniz formula and the unity of mathematics. 12 Edinstvo matematiki.pdf, 12 p. (in Russian).

[25] Hawking D., Blakeslee S. On intelligence. Translation from English. M.: ID Williams LLC, (2007). - 240 p. (in Russian).

[26] Davidson R.J., Begley S.: How emotions control the brain. Change your emotions and you change your life. - SPb.: Peter, (2012). - 256 p. (in Russian). 